\title{
A Interrupção da Gravidez na Adolescência: aspectos epidemiológicos numa maternidade pública no nordeste do Brasil'
}

\section{The Interruption of Pregnancy Amongst Adolescents: epidemiologic aspects in a public (stately run) maternity in}

northeast Brazil

\author{
José Humberto Belmino Chaves \\ Doutor em Bioética pela Universidade do Porto, Portugal. Professor \\ de Ginecologia da Universidade Federal de Alagoas e da Univer- \\ sidade Estadual de Ciências da Saúde de Alagoas. \\ Endereço: Universidade Federal de Alagoas, Faculdade de Medi- \\ cina, Campus A. C. Simões. Av. Lourival Melo Mota, s/n, Tabuleiro \\ dos Martins, CEP 57072-970, Maceió, AL, Brasil. \\ E-mail: jhbchavesळuol.com.br \\ Leo Pessini \\ Doutor em Teologia Moral/Bioética. Professor Doutor do Centro \\ Universitário São Camilo. \\ Endereço: Rua Eng. Ranulpho Pinheiro Lima, 259, CEP 04264-030, \\ São Paulo, SP, Brasil. \\ E-mail: pessineœscamilo.edu.br

\section{Antônio Fernando de Sousa Bezerra} \\ Doutor em Anatomia patológica. Professor Doutor da Universidade \\ Federal de Alagoas e da Universidade Estadual de Ciências da \\ Saúde de Alagoas. \\ Endereço: Rua Orlando Pugliese, 78 Ap. 02, Gruta de Lourdes, CEP \\ 57052-614, Maceió, AL, Brasil. \\ E-mail: afsbıзळgmail.com

\section{Guilhermina Rego} \\ Doutora em Ciências Empresariais. Professora Doutora da Facul- \\ dade de Medicina da Universidade do Porto. \\ Endereço: Faculdade de Medicina da Universidade do Porto, Ala- \\ meda Prof. Hernâni Monteiro, CEP 4200-319, Porto, Portugal. \\ E-mail: egsaudeळmed.up.pt
}

\section{Rui Nunes}

Doutor em Bioética. Professor Catedrático da Faculdade de Medicina da Universidade do Porto.

Endereço: Faculdade de Medicina da Universidade do Porto, Alameda Prof. Hernâni Monteiro, CEP 4200-319, Porto, Portugal.

E-mail: ruinunesळmed.up.pt

I Trabalho realizado no âmbito do Programa Doutoral em Bioética pela Faculdade de Medicina da Universidade do Porto, Porto, Portugal e Conselho Federal de Medicina, Brasilia, Brasil.

\section{Resumo}

O abortamento provocado é praticado amplamente pelas mulheres, em contexto clandestino, no Brasil, sendo considerado uma questão de saúde pública. Neste estudo, propõe-se a conhecer aspectos socio-demográficos, comportamentais, clínicos, complicações, e o tipo de abortamento praticado por adolescentes submetidas à curetagem uterina. Foi utilizada metodologia descritiva, através de entrevistas por meio de questionário estruturado no atendimento a 201 adolescentes com abortamento incompleto submetidas à curetagem uterina, em um hospital do Sistema Único de Saúde (SUS), em Maceió, Alagoas. Os principais determinantes para o abortamento foram: idade acima de dezesseis anos, com parceiro estável; pardas; não planejaram a gestação; desejavam a gestação, primigestas; idade gestacional menor que 15 semanas; raras complicações relacionadas ao abortamento, e utilizando a classificação da Organização Mundial da Saúde, observou-se abortamento provocado em $98,01 \%$ dos casos. Entre os casos de abortamentos certamente provocados, $89,19 \%$ reportaram o uso do misoprostol, o que reforça maior investimento público na assistência ao uso de métodos contraceptivos entre os adolescentes respeitando seus direitos sexuais e reprodutivos.

Palavras-chave: Abortamento; Aborto provocado; Adolescente; Epidemiologia; Saúde reprodutiva. 


\section{Abstract}

The induced abortion is widely practiced by women in the context illegal in Brazil, considered a public health issue. To understand the complications and the clinical, behavioral and socio-demographic aspects and type of abortion in adolescents undergoing uterine curettage. A descriptive method based on interviews using a structured questionnaire related to the care of 201 adolescents with incomplete abortion and that underwent curettage in a hospital of the Health System, Maceió - Alagoas. Adolescents at risk of causing abortion are over sixteen, brown, with a stable partner, with either a wanted or an unwanted first pregnancy, gestational age less than 15 weeks; rare complications related to abortion, and the classification of World Health Organization, induced abortion in $98.01 \%$ of cases. Among the certainly caused miscarriages, $89.19 \%$ reported the use of misoprostol, which enhance greater public investment in assisting the use of contraceptives among adolescents by respecting their sexual and reproductive rights.

Keywords: Abortion; Adolescent; Epidemiology; Provoked Abortion; Reproductive Health.

\section{Introdução}

A Organização Mundial da Saúde define como adolescentes as pessoas com idades entre 10 e 19 anos, definição adotada no Brasil pelo Programa de Saúde do Adolescente do Ministério da Saúde (Correa, 2003). A população brasileira de adolescentes atualmente é de cerca de 34.157.631 milhões de indivíduos, correspondendo a 18,6\% dos habitantes do País (IBGE, 2009).

No Brasil, ainda que lentamente, o ritmo de queda no número de partos na adolescência vem acontecendo nos últimos anos na rede pública. Dados mais recentes do Ministério da Saúde mostram que a quantidade desses procedimentos em adolescentes de 10 a 19 anos caiu 22,4\% de 2005 a 2009. Na primeira metade da década passada, a redução foi de 15,6\%. De 2000 a 2009, a maior taxa de queda anual ocorreu no ano passado, quando foram realizados 444.056 partos em todo o País - 8,9\% a menos que em 2008. Em 2005, foram registrados 572.541. Ao longo da década, a redução total foi de 34,6\% (DATASUS, 2010).

O Ministério da Saúde atribui essa tendência às campanhas destinadas aos adolescentes e à ampliação do acesso ao planejamento familiar. Só em 2009, foram investidos R\$ 3,3 milhões nas ações de educação sexual e reforço na oferta de preservativos aos jovens brasileiros. Nos últimos dois anos, 871,2 milhões de camisinhas foram distribuídos para toda a população (Brasil, 2010).

No entanto, dados oficiais relatam que no Brasil o aborto ocorre em 31\% das gestações em mulheres de 15 a 49 anos. Assevera-se a estimativa de 1,4 milhões de abortos clandestinos por ano no Brasil (Mariutti e Boemer, 2003). Comparado a países da Europa Ocidental, onde o aborto é realizado de maneira legal e acessível, esses números se revelam desastrosos na vivência dessas mulheres (Souza e col., 2001).

Como reflexo dessa situação, a curetagem pósabortamento representa o segundo procedimento obstétrico mais realizado, nas unidades de internação da rede pública de serviços de saúde, superada apenas pelos partos normais (Brasil, 2005). Dados do Ministério da Saúde - Sistema de Informações Hospitalares do Sistema Único de Saúde revelam 
registro de janeiro de 2010 a março de 2011, uma frequência de 45.342 procedimentos de curetagens pós-aborto, em mulheres abaixo de 19 anos (SIHDATASUS, 2010).

O abortamento ou interrupção da gravidez é considerado espontâneo quando se inicia independentemente de qualquer procedimento ou mecanismo externo, geralmente devido a problemas de saúde da mulher ou do feto. É considerado provocado, quando resulta da utilização de qualquer processo abortivo externo, químico ou mecânico. Este último pode ter motivação voluntária ou involuntária da gestante, e ser considerado legal ou ilegal (Blumenthal, 2003).

O Código Penal Brasileiro, promulgado em 1940, prevê somente duas situações em que o aborto é considerado legal: se for a única forma de salvar a vida da mulher grávida ou se a gravidez foi resultado de um estupro ou incesto. Além disso, em alguns casos de má-formação congênita é previsto autorização do abortamento (AADS, 2003).

Um importante problema é a falta de identificação dos casos de abortamentos ilegais, ainda mais que os métodos utilizados para provocá-los, muitas vezes, levam a quadros infecciosos e hemorrágicos graves (Elfenbein e Felice, 2003). Tais complicações comprometem a saúde da mulher e são a causa de $10 \%$ a $15 \%$ de óbitos maternos no Brasil (Andrade e col., 2006). O coeficiente de mortalidade decorrente do aborto é 2,5 vezes maior em menores de 20 anos (Brown, 2006).

A realização de pesquisas epidemiológicas com pacientes hospitalizados, por complicações relacionadas ao abortamento tem especial relevância para o estudo da morbimortalidade materna e para estimativa dos custos direcionados ao cuidado dessa patologia (Camargo e col., 2011; Araújo e Viola, 2003).

Apesar da mortalidade em decorrência do abortamento provocado estar diminuindo, devido às mudanças nas práticas utilizadas, pois atualmente as mulheres recorrem mais ao uso de misoprostol e menos ao uso de sondas vaginais, que podem determinar maior risco de perfuração uterina, sangramento e morte, o número de complicações ainda é grande, o que justifica uma sistematização do atendimento ao abortamento provocado (Loku- gamage e col., 2003).

Nesse sentido, no Brasil, o Ministério da Saúde lançou, em março de 2005, o Manual de Normas Técnicas (Brasil, 2005), para atendimento humanizado ao abortamento nos hospitais públicos do SUS; mas existe desconhecimento por parte dos gestores de hospitais e dos profissionais dessas normas em diversas localidades do país.

0 recurso ao aborto pelas adolescentes reforça ainda mais a argumentação de que as gestações que o originaram são consideradas sempre "não planejadas” (Brindis, 2006). Assim, segundo a OMS, no Brasil, uma em cada nove mulheres recorre ao aborto como meio de pôr fim a uma gestação não planejada (OMS, 2004).

Levando em consideração a importância do quadro de abortamento para saúde física e emocional da adolescente, bem como o desenvolvimento da assistência prestada a ela, o presente estudo objetiva descrever aspectos sociodemográficos, clínicos, comportamentais, complicações da curetagem uterina, analisar o tipo de abortamento, quanto à motivação em adolescentes que foram internadas na Casa Maternal Dr. Paulo Neto em Maceió, Alagoas, para realização de curetagem uterina.

\section{Metodologia}

O Ministério da Saúde do Brasil, ao normatizar os procedimentos para o atendimento do abortamento em serviços públicos, lançou um guia geral objetivando fornecer aos profissionais subsídios para que possam oferecer não só cuidado imediato, mas também, na perspectiva da integralidade desse atendimento, oferecer alternativas contraceptivas, como uma forma de evitar o recurso ao abortamento. (Brasil, 2005)

É nesse contexto que um grupo de profissionais da saúde se motivou a realizar um estudo descritivo com adolescentes com quadro de abortamento, atendidas numa maternidade conveniada com o SUS em Maceió, Alagoas, à época referência para o procedimento de curetagem uterina, visando conhecer o problema e sensibilizar os gestores públicos a garantir integralidade da assistência e estabelecer como modelo as bases técnicas para atendimento ao 
abortamento em todo o Estado de Alagoas.

Utilizamos como técnicas de coleta de informações questionários com roteiros pré-estabelecidos, aplicados e preenchidos nas enfermarias, à beira do leito, pelos profissionais de saúde previamente treinados, com perguntas sobre informações sociodemográficas (idade, situação conjugal, cor); fatores associados ao comportamento e estilo de vida (uso de álcool, de fumo, gravidez planejada, gravidez desejada), dados clínicos (idade gestacional, número de gestações, número de abortos), e dados do procedimento de curetagem uterina (perfuração uterina, transfusão sanguínea).

Antes do início da entrevista, cada paciente foi informada sobre o objetivo do estudo e convidada a assinar o termo de consentimento livre e esclarecido para sua participação, sendo assegurado às participantes a confidencialidade das informações fornecidas. As entrevistas foram realizadas de forma privada ou acompanhada do responsável legal respeitando a autonomia da adolescente, sempre após o exame obstétrico de internamento hospitalar e antes do procedimento cirúrgico da curetagem uterina.

Das 223 adolescentes grávidas internadas com diagnóstico de interrupção da gravidez, 22 delas, por diferentes motivos, não tiveram suas entrevistas concluídas. As informações colhidas e processadas no período compreendido entre março de 2008 a abril de 2009, foram inseridas no banco de dados do Sistema de Informações Hospitalares do Sistema Único de Saúde (SIH-SUS) à época do estudo, referentes aos procedimentos e a Classificação Internacional das Doenças (CID) específicos relacionados à curetagem uterina.

Todos os casos de abortamento foram diagnosticados com base em critérios médicos préestabelecidos, bem como as respostas colhidas nas entrevistas, e tipo de abortamento, sendo classificado em quatro categorias, de acordo com a proposta da Organização Mundial da Saúde (WHO, 1987): (a) certamente provocado, quando a mulher admitiu ter provocado o aborto ou quando foram encontrados sinais clínicos de intervenção, tais como laceração cervical e/ou corpo estranho na vagina ou no útero; (b) provavelmente provocado, quando a mulher não admitiu ter provocado o aborto, mas referiu gravidez não planejada e foram encontrados sinais de sepsis ou peritonite; (c) possivelmente provocado, quando somente uma das duas condições listadas em (b) esteve presente. Todos os outros casos de abortamento foram classificados como espontâneos.

$\mathrm{Na}$ análise dos dados, aplicou-se o teste de associação do Qui-quadrado para tabela 2 x 2 (Quiquadrado com correção de Yates). Foram considerados estatisticamente significativos os valores de $p<$ o,05. O software Epi-Info foi utilizado para cálculo de frequências e tabelas.

Essa pesquisa foi realizada seguindo as normas para pesquisas envolvendo seres humanos, estabelecidas pela Resolução 196/96 do Conselho Nacional de Saúde, seguindo os princípios éticos da Declaração de Helsinki e após aprovação pelo Comitê de Ética em pesquisa da Universidade Federal de Alagoas (UFAL) (Processo nº10679/2008-51).

\section{Resultados}

Foram investigadas 201 adolescentes durante o período de estudo, de acordo com os dados especificados na Tabela 1. Considerando o grupo etário, a maior proporção de adolescentes, 88,06\%, encontrava-se na faixa dos 15 aos 19 anos de idade. Houve diferença significativa dos tipos de abortamento em relação à faixa etária. Em todos os tipos, a maior proporção ocorreu na mesma faixa de idade em que predominaram os abortos de modo geral.

Entre as adolescentes, menores que 15 anos, que representaram 11,94\% do universo estudado, o tipo de abortamento mais encontrado foi o certamente provocado $(14,02 \%)$, seguido pelos possivelmente provocados $(7,69 \%)$. Não houve, nesse grupo etário, nenhum caso de abortamento provavelmente provocado. No entanto, entre as adolescentes de 15 a 19 anos, $85,98 \%$ dos casos foram de abortamentos certamente provocados.

Com relação à situação conjugal, as adolescentes com parceiro estável estão relacionadas em maior percentual com abortamento certamente provocado, $69,51 \%$, e abortamento possivelmente provocado, $53,85 \%$. No grupo das adolescentes sem parceiro estável, os abortamentos certamente provocados apresentam valores acima do dobro do percentual, 
Tabela I - Distribuição do número e proporção (\%) de adolescentes grávidas segundo tipo de abortamento $e$ condições sociodemográficas e comportamento. Maceió, AL, 2008

\begin{tabular}{|c|c|c|c|c|c|c|c|c|c|c|c|}
\hline \multirow{3}{*}{ Variáveis } & \multicolumn{11}{|c|}{ Tipos de abortamento } \\
\hline & \multicolumn{2}{|c|}{$\begin{array}{l}\text { Certamente } \\
\text { provocado }\end{array}$} & \multicolumn{2}{|c|}{$\begin{array}{c}\text { Provavelmente } \\
\text { provocado }\end{array}$} & \multicolumn{2}{|c|}{$\begin{array}{c}\text { Possivelmente } \\
\text { provocado }\end{array}$} & \multicolumn{2}{|c|}{ Espontâneo } & \multicolumn{2}{|c|}{ Total } & \multirow[t]{2}{*}{$p^{*}$} \\
\hline & $\mathrm{N}$ & $\%$ & $\mathrm{~N}$ & $\%$ & $\mathrm{~N}$ & $\%$ & $\mathrm{~N}$ & $\%$ & $\mathrm{~N}$ & $\%$ & \\
\hline \multicolumn{12}{|l|}{ Cor/raça } \\
\hline Preta & 8 & 4,8 & 0 & 0 & 0 & 0 & 0 & 0 & 8 & 3,9 & \multirow{5}{*}{ ns } \\
\hline Parda & 103 & 62,8 & 9 & 45,0 & 8 & 61,5 & 1 & 25,0 & 121 & 60,2 & \\
\hline Branca & 50 & 30,5 & 9 & 45,0 & 3 & 23,1 & 3 & 75,0 & 65 & 32,4 & \\
\hline Amarela & 3 & 1,9 & 2 & 10,0 & 2 & 15,4 & - & - & 7 & 3,50 & \\
\hline Indigena & - & - & - & - & - & - & - & - & - & - & \\
\hline \multicolumn{12}{|c|}{ Situação conjugal } \\
\hline Sem parceiro & 50 & 30,4 & 14 & 70,0 & 6 & 46,1 & 2 & 50,0 & 72 & 35,8 & \multirow{2}{*}{ ns } \\
\hline Com parceiro & 114 & 69,5 & 6 & 30,0 & 7 & 53,8 & 2 & 50,0 & 129 & 64,1 & \\
\hline \multicolumn{12}{|l|}{ Álcool } \\
\hline Sim & 20 & 12,2 & - & - & 3 & 23,0 & - & - & 23 & 11,4 & \multirow{2}{*}{ ns } \\
\hline Não & 144 & 87,8 & 20 & 100,0 & 10 & 76,9 & 4 & 100,0 & 178 & 88,5 & \\
\hline \multicolumn{12}{|l|}{ Fumo } \\
\hline Sim & 30 & 18,2 & 2 & 10,0 & 2 & 15,3 & - & - & 34 & 16,9 & \multirow{2}{*}{ ns } \\
\hline Não & 134 & $8 \mathrm{I}, 7$ & 18 & 90,0 & ॥ & 84,6 & 4 & 100,0 & 167 & 83,0 & \\
\hline Total & 164 & 81,5 & 20 & 9,9 & 13 & 6,4 & 4 & 1,9 & 201 & 100,0 & \\
\hline
\end{tabular}

${ }^{*} p$, ns = não significante (teste $\left.\chi^{2}\right)$.

e o abortamento provavelmente provocado, foi o tipo mais prevalente (70,00 \%).

Quanto ao critério cor/raça das adolescentes, a maior prevalência de abortamento provocado foi verificada em mulheres pardas $(60,20 \%)$, seguidas de brancas $(32,4 \%)$, pretas $(3,98 \%)$ e amarelas $(3,50 \%)$.

Quanto ao uso de álcool e fumo, a maioria das adolescentes, em todas as categorias do abortamento, não fazia uso de álcool ou de fumo.

A Tabela 2 mostra dados da distribuição de gestantes adolescentes, segundo idade gestacional e antecedentes reprodutivos. Em 164 adolescentes de abortamento certamente provocado, a idade gestacional em que ocorreu a perda gestacional, em $85,98 \%$ das vezes, foi antes de 15 semanas, isto é, na fase de abortamento tardio. Com relação ao número de gestação, entre as mulheres com abortamento provocado, o maior percentual foi de primigestas.

Quanto à ocorrência de aborto prévio, verificouse que a maioria das mulheres do estudo não tinha história anterior de abortamento. Entre os casos classificados como abortamentos provocados, em $10,94 \%$ houve relato de um aborto anterior a essa gestação; em 2,49\%, dois abortos; em nenhuma, três, quatro ou mais abortos. Dos casos com diagnóstico de abortamentos espontâneos, 100\% referiam não ter aborto anterior.

A Tabela 3 mostra a distribuição de adolescentes grávidas, segundo o planejamento e o desejo de engravidar. A proporção daquelas que desejaram e planejaram $(97,26 \%)$ foi maior do que aquelas que desejaram, mas não planejaram (59,38\%); essa diferença é estatisticamente significante, Qui-Quadrado 
Tabela 2 - Distribuição do número e proporção (\%) de adolescentes grávidas segundo tipo de abortamento e antecedentes reprodutivos, Maceió, AL, 2008

\begin{tabular}{|c|c|c|c|c|c|c|c|c|c|c|c|}
\hline \multirow{3}{*}{ Variáveis } & \multicolumn{11}{|c|}{ Tipos de abortamento } \\
\hline & \multicolumn{2}{|c|}{$\begin{array}{l}\text { Certamente } \\
\text { provocado }\end{array}$} & \multicolumn{2}{|c|}{$\begin{array}{c}\text { Provavelmente } \\
\text { provocado }\end{array}$} & \multicolumn{2}{|c|}{$\begin{array}{c}\text { Possivelmente } \\
\text { provocado }\end{array}$} & \multicolumn{2}{|c|}{ Espontâneo } & \multicolumn{2}{|c|}{ Total } & \multirow[t]{2}{*}{$p^{*}$} \\
\hline & $n$ & $\%$ & $n$ & $\%$ & $n$ & $\%$ & $n$ & $\%$ & $n$ & $\%$ & \\
\hline \multicolumn{12}{|c|}{ Idade gestacional } \\
\hline 10 & - & - & - & - & - & - & - & - & - & - & \multirow{4}{*}{ ns } \\
\hline $11-12$ & 11 & 6,71 & 0 & 0 & - & - & - & - & 11 & 5,47 & \\
\hline $13-15$ & 141 & 85,98 & 20 & 100 & 12 & 92,31 & 4 & 100 & 141 & 85,98 & \\
\hline $16 e+$ semanas & 12 & 7,32 & - & - & 1 & 7,69 & - & - & 13 & 6,47 & \\
\hline \multicolumn{12}{|c|}{ Número de gestação } \\
\hline 1 & 85 & 51,83 & 12 & 60 & 6 & 46,15 & 2 & 50 & 105 & 52,24 & \multirow{4}{*}{ ns } \\
\hline 2 & 50 & 30,49 & 4 & 20 & 5 & 38,46 & 2 & 50 & 61 & 30,35 & \\
\hline 3 & 20 & 12,20 & 4 & 20 & 2 & 15,38 & - & - & 26 & 12,94 & \\
\hline $4^{+}$ & 9 & 5,49 & - & - & - & - & - & - & 9 & 4,48 & \\
\hline \multicolumn{12}{|c|}{ Número de partos } \\
\hline 0 & 20 & 12,20 & 6 & 30 & 1 & 7,69 & 2 & 50 & 29 & 14,43 & \multirow{4}{*}{ ns } \\
\hline 1 & 70 & 42,68 & 6 & 30 & 5 & 38,46 & 2 & 50 & 83 & 41,29 & \\
\hline 2 & 45 & 27,44 & 4 & 20 & 5 & 38,46 & - & - & 54 & 26,87 & \\
\hline 3 & 20 & 12,20 & 4 & 20 & 2 & 15,38 & - & - & 26 & 12,94 & \\
\hline \multicolumn{12}{|l|}{ Aborto anterior } \\
\hline 0 & 144 & 87,80 & 14 & 70 & 12 & 92,31 & 4 & 100 & 174 & 86,57 & \multirow{5}{*}{ ns } \\
\hline 1 & 15 & 9,15 & 6 & 30 & 1 & 7,69 & - & - & 22 & 10,95 & \\
\hline 2 & 5 & 3,05 & - & - & - & - & - & - & 5 & 2,49 & \\
\hline 3 & - & - & - & - & - & - & - & - & - & - & \\
\hline $4^{+}$ & - & - & - & - & - & - & - & - & - & - & \\
\hline Total & 164 & 100 & 20 & 100 & 13 & 100 & 4 & 100 & 201 & 100 & \\
\hline
\end{tabular}

${ }^{*} p$, ns $=$ não significante $\left(\right.$ teste $\left.\chi^{2}\right)$.

corrigido por Yates de 34,42 ( $p=0,00000001)$.

Com relação aos métodos utilizados para indução do abortamento, das 164 adolescentes classificadas na categoria abortamento certamente provocado, a maioria, 89,19\%, relatou, na indução do abortamento, o uso de misoprostol de forma isolada, $20,12 \%$, ou em combinação com outros métodos, $77,44 \%$.

Entre as adolescentes submetidas ao procedimento de curetagem uterina, houve três casos de perfuração, isto é, 1,49\%, sendo que em um caso o abortamento foi certamente provocado. Entre os casos com perfuração uterina, dois casos apresentaram abortamento provavelmente provocado, não havendo caso de perfuração no abortamento espontâneo.

A transfusão sanguínea foi realizada em nove casos, sendo que em oito o abortamento foi certamente provocados. Desses, $11,11 \%$ dos casos tiveram abortamento classificado como provavelmente provocado, e nenhum abortamento espontâneo. 
Tabela 3 - Distribuição do número e proporção (\%) de adolescentes grávidas segundo tipo de abortamento e o planejamento e o desejo de engravidar. Maceió, AL, 2008

\begin{tabular}{|c|c|c|c|c|c|c|c|c|c|c|c|}
\hline \multirow{3}{*}{ Variáveis } & \multicolumn{11}{|c|}{ Tipos de abortamento } \\
\hline & \multicolumn{2}{|c|}{$\begin{array}{l}\text { Certamente } \\
\text { provocado }\end{array}$} & \multicolumn{2}{|c|}{$\begin{array}{c}\text { Provavelmente } \\
\text { provocado }\end{array}$} & \multicolumn{2}{|c|}{$\begin{array}{c}\text { Possivelmente } \\
\text { provocado }\end{array}$} & \multicolumn{2}{|c|}{ Espontâneo } & \multicolumn{2}{|c|}{ Total } & \multirow[t]{2}{*}{$p^{*}$} \\
\hline & $\mathrm{N}$ & $\%$ & $\mathrm{~N}$ & $\%$ & N & $\%$ & $\mathrm{~N}$ & $\%$ & $\mathrm{~N}$ & $\%$ & \\
\hline \multicolumn{12}{|c|}{ Gravidez planejada } \\
\hline $\operatorname{sim}$ & 68 & 41,4 & 4 & 20,0 & 1 & 7,6 & - & - & 73 & 36,3 & \multirow{2}{*}{ ns } \\
\hline Não & 96 & 58,5 & 16 & 80,0 & 12 & 92,3 & 4 & 100,0 & 128 & 63,6 & \\
\hline \multicolumn{12}{|c|}{ Gravidez desejada } \\
\hline Sim & 140 & 85,3 & 4 & 20,0 & 1 & 7,6 & 2 & 50,0 & 147 & 73,1 & \multirow{2}{*}{ ns } \\
\hline Não & 24 & 14,6 & 16 & 80,0 & 12 & 92,3 & 2 & 50,0 & 54 & 26,8 & \\
\hline \multicolumn{12}{|c|}{ Gravidez planejada e desejada } \\
\hline $\operatorname{sim}$ & - & - & - & - & - & - & - & - & 195 & 97,2 & \multirow{2}{*}{$<0,001$} \\
\hline Não & - & - & - & - & - & - & - & - & 6 & 2,8 & \\
\hline \multicolumn{12}{|c|}{ Gravidez desejada, mas não planejada } \\
\hline $\operatorname{sim}$ & - & - & - & - & - & - & - & - & 119 & 59,3 & \multirow{2}{*}{$<0,001$} \\
\hline Não & & & & & & & & & 82 & 40,7 & \\
\hline Total & 164 & 81,5 & 20 & 9,9 & 13 & 6,4 & 4 & 1,9 & 201 & 100,0 & \\
\hline
\end{tabular}

${ }^{*} p$, ns = não significante (teste $\left.\chi^{2}=32,4\right)$.

\section{Discussão}

Através deste instrumento preconizado pela Organização Mundial da Saúde (WHO, 1987), observouse que, quanto à motivação para a prática do abortamento entre as adolescentes do presente estudo, 1,99\% tiveram abortamento espontâneo e o restante, 98,o1\% abortamentos provocados. Entre estes, verificou-se que em $81,59 \%$ dos casos, havia evidências de que a interrupção da gravidez havia sido provocada.

A adolescência tem sido, nas últimas décadas, motivo de grande preocupação, principalmente entre a faixa etária de 15 a 19 anos, visto que além de se constituir 8,83\% (16.936) da população brasileira (IBGE, 2009), acompanha-se quase sempre de condições insatisfatórias e inadequadas de conhecimentos sobre vida sexual e reprodutiva (Villela e col., 2007).

Além disso, alguns autores (Taquette e col., 2005; Rasch, 2003) observaram; que o número de mulheres que buscaram atendimento hospitalar para o tratamento do abortamento certamente provocado um percentual significativo é constituído por adolescentes. Corroborando esses dados, em estudo prospectivo de Bailey e colaboradores (2001); observou em 196 adolescentes com abortamento incompleto que em $66,7 \%$ dos casos o abortamento era provocado.

Trata-se, portanto, de um cenário desfavorável para as adolescentes, pois diante de uma relação sexual desprotegida só lhes resta o recurso do aborto quando uma gravidez não prevista ocorre.

O Congresso Internacional sobre População e Desenvolvimento (CIPD), reconhecendo a temática do abortamento como um problema de saúde pública, solicitou aos governos e às organizações intergovernamentais e não governamentais a redução do recurso das mulheres ao aborto através de serviços de melhor planejamento familiar, informações viáveis e aconselhamento (Faúndes e col., 2009).

De acordo com este estudo, das adolescentes que buscaram atendimento hospitalar, 69,51\% tinham parceiro estável e em sua maioria não eram usuárias de álcool e tabaco, 88,56\% e 83,08\%, respectivamente. Portanto, existe consenso na literatura que o uso abusivo de álcool (Rasch, 2003) e de tabaco (Ness e col., 1999) aumenta o risco de ocorrência de abor- 
tamento espontâneo, possivelmente por alterações de vascularização da decídua; entretanto parece não haver estudos relacionando esses hábitos à prática do abortamento provocado principalmente em adolescentes.

Neste estudo, o maior percentual de abortamento provocado foi registrado na primeira gestação e com idade gestacional abaixo de 15 semanas, número semelhante ao referido por outros estudos em países latino-americanos (Souza e col., 2001; Brasil, 2009).

Diferentemente, Souza e colaboradores (2001), observaram que a variável paridade em um grupo de adolescentes com abortamentos provocados tiveram proporção elevada nas que reportaram ter mais de um filho vivo, o que poderia sugerir, que as adolescentes, de maneira geral, procuram o aborto após a primeira gestação.

Quanto à ocorrência de aborto prévio, verificouse que $10,95 \%$ das adolescentes que abortaram relataram história de aborto anterior. Outros autores (Griebel e col., 2005), verificaram que 20,1\% das adolescentes relataram aborto anterior.

Dentre as adolescentes com interrupção da gravidez supostamente provocada, $128(63,68 \%)$ não haviam planejado a gravidez e 147 (73,13\%) não a haviam desejado. Do mesmo modo, pesquisa realizada em outras capitais brasileiras o recurso ao abortamento, ao ser identificado nas adolescentes, reforça ainda mais a argumentação de que as gestações que o originaram são consideradas eventos sempre "não planejados" (Brasil, 2oog).

Do mesmo modo, a pesquisa sobre Saúde Reprodutiva e Sexualidade dos Jovens, realizada por meio de inquérito domiciliar em outras capitais brasileiras (Farias Júnior e col., 2009); revelou altos percentuais de gravidezes consideradas não planejadas, de $46 \%$ a $58 \%$.

Dessa forma, as gestantes adolescentes apresentaram uma forte associação entre gravidez desejada e não planejada, ou seja, nessa fase da vida a adolescente quer fortemente ser mãe, mas possivelmente não tenha maturidade para o devido planejamento de todos os elementos consequentes à maternidade precoce. Ao que parece, no Brasil, o aborto ainda é largamente utilizado pelas mulheres como uma solução para a gravidez não planejada, especialmente em ambientes que dificultam o acesso aos contraceptivos eficazes (Farias Júnior e col., 2009).

De acordo com os nossos resultados, o abortamento provocado acontece em adolescentes sem filhos, com parceiros estáveis e que não planejaram a gravidez. A literatura sobre o fenômeno da gravidez na adolescência (Faúndes e col., 2009; Souza e col., 2001); apontam que esse problema poderia ser resolvido através de um programa de contracepção facilmente acessível às adolescentes que buscam no aborto um modelo para evitar a gravidez não planejada.

Adicionalmente, a ausência de autonomia material e financeira dos jovens e o engajamento em relações ainda não consolidadas contrariam a representação dominante que valoriza a chegada do filho sob certas condições (Griebel e col., 2005). Esses fatores são mais importantes na decisão pelo abortamento do que a possibilidade de complicações decorrentes desse procedimento, incluindo a morte (Woolf e Maisto, 2008).

De fato, a saúde sexual e reprodutiva dos adolescentes é motivo de constante preocupação, uma vez que suas consequências são de alto impacto individual e social (Griebel e col., 2005).

Apesar da dificuldade de nortear as práticas sexual e reprodutiva na adolescência, pesquisas realizadas em diferentes países têm verificado a interferência de distintos fatores no aumento da fecundidade nessa população, destacando-se a iniciação sexual precoce associada ao desconhecimento sobre saúde reprodutiva e a pouca utilização de contraceptivos, seja por falta de orientação da família e da escola ou pela ineficiência de serviços de planejamento familiar (Farias Júnior e col., 2009).

Em nosso estudo, o abortamento foi certamente provocado em $89,19 \%$ pelo uso de misoprostol. Esse medicamento, comercializado desde o início da década de 1990, com indicação de tratamento para doença péptica, mostrou-se eficiente na indução do abortamento, tornando-se popularmente conhecido para esse fim. Isso contribui consideravelmente na redução das complicações infecciosas em pacientes com abortamento provocado (Griebel e col., 2005).

Blum e colaboradores (2007), numa revisão da literatura conduzida para determinar se o misoprostol é um tratamento eficaz para o aborto incompleto, 
concluíram ser a melhor escolha principalmente quando o tamanho uterino for equivalente a 12 semanas de gestação. Em estudo com 1.456 mulheres com quadro de abortamento (Kapil Ahmed e col., 2005), constatou-se que a utilização do misoprostol foi o método de abortamento utilizado.

Entretanto, publicações nacionais numa coletânea de duas décadas (Brasil, 2009) registram que ainda permanece desconhecido o número de casos em que as adolescentes iniciam o aborto em casa com misoprostol e terminam em hospitais. Não se sabe também se há mulheres que começam e finalizam o aborto em casa utilizando esse método. $\mathrm{Na}$ verdade, a casuística que conhecemos e aquela que chega aos hospitais públicos.

Este medicamento não é adquirido com facilidade, pois a forma de compra em balcão de farmácia exige o receituário médico, portanto observa-se que na prática do abortamento provocado o acesso a compra do medicamento é feito de forma clandestina.

Com a clandestinidade, o aborto é considerado uma das principais causas de mortalidade (AADS, 2003), pois percebemos que nas situações em que o aborto provocado é crime surgem os tipos de aborto descrito na lei, que fala sobre a mulher vítima de estupro e ou quando a gestante corre risco de morrer.

O número de casos de abortamentos provocados com sequelas graves e o coeficiente de letalidade têm apresentado acentuada diminuição (Blum e col., 2007). É possível que isso esteja relacionado ao crescente uso de misoprostol, como método abortivo, por mulheres de baixa renda.

No Brasil, sabe-se que a maioria dos abortamentos são praticados não em hospitais, mas sim em locais inseguros, que precisam se manter em anonimato, determinando que as mulheres que apresentam complicações pós-abortamento, como hemorragia, infecção etc. possam ser internadas não nas maternidades, mas em hospitais gerais, como portadoras de patologias sem relação com o processo de abortamento, o que piora os índices de sub-registro (Brasil, 2009).

Investigações sobre casos de mulheres que desenvolveram condições mórbidas graves por causas maternas, mas que sobreviveram, e os poucos trabalhos identificados no país com esse recorte assinalam a importância dessa vertente de análise na obtenção de dados mais acurados sobre essas condições e avaliação da qualidade da assistência obstétrica (Farias Júnior e col., 2009).

0 mesmo já não se pode afirmar em relação à mortalidade de adolescentes em consequência do aborto. Embora não existam estatísticas nacionais confiáveis, várias investigações sobre a mortalidade materna (morte de mulheres durante a gestação, parto e puerpério, exceto as decorrentes de eventos acidentais ou incidentais) têm avaliado qual o papel desempenhado pelo aborto na gênese dessa mortalidade (AADS, 2003).

Em todos os casos selecionados para este estudo, o tratamento de escolha foi a curetagem uterina para remoção dos restos placentários. Nesse sentido, de acordo com Griebel e colaboradores (2005) e Araújo e Viola (2003), a curetagem uterina, indicada para o esvaziamento uterino por abortamento incompleto, é o segundo procedimento obstétrico mais realizado nos hospitais do Sistema Único de Saúde, superado apenas por partos normais. Ele é o método mais realizado no tratamento do aborto na grande maioria dos hospitais da rede pública do Brasil (Brasil, 2009).

O abortamento é uma prática comum em todas as classes sociais, idades e estado civil, porém, dependendo da situação financeira, os riscos são maiores ou menores, assinalando a diferença entre a adolescente de maior e de menor poder aquisitivo (Araújo e Viola, 2003). Esses aspectos, todavia, não expressam a complexa relação das adolescentes com a interrupção da gravidez.

Neste estudo, não houve impedimento da participação de acompanhante na entrevista, apesar de estar presente na metodologia a possibilidade da presença de acompanhante, considerando tratarse de uma população de adolescente, em nenhuma entrevista ocorreu a presença de acompanhante, eliminando assim a possibilidade de viés de informação em função de sua presença.

A presença de um acompanhante na hora da coleta do questionário pode interferir na veracidade das informações colhidas (Brasil, 2005). Além de as maternidades apresentarem espaços mínimos para esse tipo de atendimento, garantindo pouca privacidade, o que expõe as adolescentes, muitas vezes, a situações constrangedoras. 
Outros estudos (Farias Júnior e col., 2009) apontam o fato de as adolescentes não serem informadas sobre os cuidados requeridos pós-procedimento, inclusive a necessidade de métodos de planejamento familiar disponíveis e da contracepção pós-aborto.

Embora a contracepção seja uma das ações previstas nas políticas de saúde desde a década de 1980, e conste como direito na Constituição Brasileira desde a mesma década, a implementação heterogênea das ações no país não assegura a efetividade de sua distribuição.

O aborto torna-se, então, a única saída para essas adolescentes e, nesse desafio, elas arriscam suas próprias vidas, quando decidem interromper a gravidez utilizando-se de pessoas não habilitadas e métodos abortivos rudimentares. Essa decisão muitas vezes é vivida de forma solitária e clandestina, ou sobre pressão dos parceiros ou familiares.

Os aspectos assinalados, ainda que importantes, não traduzem por si só a complexa relação das adolescentes com os profissionais da saúde que prestam assistência ao atendimento do abortamento em serviço público, principalmente na perspectiva da integralidade.

Diante do que foi explicitado, este estudo permite traçar o perfil de adolescentes com risco de provocarem o abortamento: idade média de 16,1 anos, maioria parda, primigesta, gravidez não planejada, porém desejada. Assim sendo, os resultados confirmam a necessidade de uma maior atenção à assistência e ao planejamento familiar, como também a implementação de programas que, efetivamente, permitam o controle da gravidez na adolescência.

\section{Referências}

ANDRADE, A. T. L. et al. Mortalidade materna: 75 anos de observação em uma Maternidade Escola. Revista Brasileira de Ginecologia e Obstetrícia, São Paulo, v. 28, n. 7, p. 380-387, 2006.

ARAÚJO, M. J. O.; VIOLA, R. C. Impacto da gravidez indesejada na saúde da mulher. In: PITANGUY, J.; MOTA, A. (Orgs.). Os novos desafios da responsabilidade política. Rio de Janeiro: CEPIA, 2005.
BAILEY, P.E. et al. Adolescent pregnancy 1 year later: the effects of abortion vs. motherhood in Northeast Brazil. Jornal Adolescent Health, New York, v. 29, p. 223-232, 2001.

BRASIL. Ministério da Saúde. Norma técnica para atenção humanizada ao abortamento. Brasília, DF, 2005.

BRASIL. Ministério da Saúde. 20 anos de pesquisas sobre aborto no Brasil. Brasília DF, 2009.

BRASIL. Ministério da Saúde. Brasil acelera redução de gravidez na adolescência. Brasília DF, 2010. Disponível em: <http://portal.saude. gov.br/portal/aplicacoes/noticias/default. cfm?pg=dspDetalheNoticia\&id_area $=124 \& \mathrm{CO}_{-}$ NOTICIA=11137>. Acesso em: 26 Abr. 2011.

BRINDIS, C. D. A public health success: understanding policy changes related to teen sexual activity and pregnancy. Annual Review of Public Health, Palo Alto, US, v. 27, p. 277-295, 2006.

BROWN, R.T. Pregnancy and abortion in adolescents. Pediatric Endocrinology Review, Netanya, v. 3, n. 1, p. 167-169, 2006.

BLUMENTHAL, P. D. Abortion: epidemiology, sufely, and techique. Current Opinion in Obstetrics \& Gynecology, Philadelphia, v. 4, n. 4, p. 506 - 512, 2003.

BLUM, B. et al. Treatment of incomplete abortion and miscarriage with misoprostol. International Journal Gynaecology Obstetrics, Baltimore, US, v. 99, p. 186-189, 2007.

CAMARGO, R. S. et al. Severe maternal morbidity and factors associated with the occurrence of abortion in Brazil. International Journal of Gynaecology and Obstetrics, Baltimore, US, v. 112, p. 88-92, 2011.

CORREA, H. Aspectos sociodemográficos sobre a maternidade na adolescência: o contexto brasileiro. Femina, Rio de janeiro, v. 31, n. 8, p. 691$695,2003$. 
DATASUS. Departamento de informática do sistema único de Saúde. MS/SVS/DASIS - Sistema de Informações sobre Nascidos Vivos - SINASC. Brasília, DF, 2010. Disponível em: http://tabnet. datasus.gov.br/cgi/tabcgi.exe?sinasc/cnv/nvuf.def. Acesso em: 2 mar. 2011.

ELFENBEIN, D. S.; FELICE, M. E. Adolescent pregnancy. Pediatric Clinics of North America, Elsevier, Philadelphia, US, v. 5o, n. 4, p. 781-786, 2003.

FARIAS JÚNIOR, J. C. et al. Comportamentos de risco à saúde em adolescentes no Sul do Brasil: prevalência e fatores associados. Revista Panamericana de Salud Pública, Washington, v. 25, n. 4, p. 34-52, 2009.

FAÚNDES, A.; RAO, K.; BRIOZZO, L. Right to protection from unsafe abortion and postabortion care. International Journal of Gynecology \& Obstetrics, Baltimore, US, v. 106, n. 2, p. 164-167, 2009.

GRIEBEL, C.P. et al. Management of spontaneous abortion. American Family Physician, Kansas City, v. 72, n. 7, p. 1243-1250, 2005.

AADS - Ações Afirmativas em Direitos e Saúde | Ipas Brasil. Mortalidade materna, Rio de Janeiro, 2003. Disponível em: http://www.aads.org.br/ wp/?page_id=1119. Acesso em: 12 jun. 2009.

IBGE - Instituto Brasileiro de Geografia e Estatística. Brasília, 2009. Tabela 261. Disponível em: http://www.sidra.ibge.gov.br/bda/desc/desc. asp? $z=$ pnad \& $0=3 \& \mathrm{i}=\mathrm{P} \& \mathrm{e}=\mathrm{c} \& \mathrm{c}=261$. Acesso em: 05 mar. 2011.

KAPIL AHMED, M.; VAN GINNEKEN, J.;

RAZZAQUE, A. Factors associated with adolescent abortion in a rural area of Bangledesh. Tropical Medicine Internacional Health, Oxford, v. 10, n. 2, p. 198-205, 2005 .

LOKUGAMAGE, A. V.; REFAEY, H. E.; RODECK, C. H. Misoprostol and pregnancy: everincreasing indication of effective usage. Current Opinion in Obstetrics and Gynecology, Philadelphia, v. 15, n. 6, p. 513-518, 2003.

MARIUTTI, M. G.; BOEMER, M. R. A mulher em situação de abortamento - um enfoque vivencial. Revista da Escola de Enfermagem da Universidade de São Paulo, São Paulo, v. 2, n. 37, p. 59-71, 2003.
NESS, R. B. et al. Cocaine e tobacco use and the risk of spontaneous abortion. New England Journal of Medicine, Boston, US, v. 340, n. 5, p. 333339, 1999.

OMS - ORGANIZAÇÃO MUNDIAL DA SAÚDE. Abortamento seguro: orientação técnica e de políticas para os sistemas de saúde. Genebra: OMS, 2004 .

RASCH, V. Cigarette, alcohol, and caffeine consumption:risk factors for spontaneous abortion. Acta Obstetric and Gynecology, Scandinavica, v. 82, n. 2, p. 182-188, 2003.

SIH-DATASUS - Sistema de Informações Hospitalares do Sistema Único de Saúde. Departamento de Informação do Sistema Único de Saúde. Infodatasus, Brasília, 2010. Disponível em: <http://formsus.datasus.gov.br/site/formulario. php?id_aplicacao=652>. Acesso em: 26 mar. 2011.

SOUZA, V. L. C. et al. O aborto entre adolescentes. Revista Latino-americana de Enfermagem, São Paulo, v. 9, n. 2, p. 42-47, 2001.

TAQUETTE, S. R. et al. Conflitos éticos no atendimento à saúde de adolescentes. Cadernos de Saúde Pública, Rio de Janeiro, n. 21, p. 1717-1725, 2005 .

WHO - WORLD HEALTH ORGANIZATION. Protocol for hospital based descriptive studies of mortality, morbidity related to induced abortion, WHO Project $n^{\circ} .86912$. Task force on safety and efficacy of fertility regulating methods. Revised edition. Geneva: WHO, 1987.

WOOLF, S. E.; MAISTO, S. A. As diferenças de gênero no comportamento do uso do preservativo? O papel do poder e sócio-tipo. Sex Roles, New York, v. 58, n. 9-10, p. 689-701, 2008.

VILLELA, W. V.; LAGO, T. Conquistas e desafios no atendimento das mulheres que sofreram violência sexual. Cadernos de Saúde Pública, Rio de Janeiro, v.23, n.2, p. 471-475. 2007.

Recebido em: 19/04/2010

Reformulado em: 15/06/2011

Aprovado em: 21/06/20II 